\title{
Renal function after nephron-sparing surgery for renal tumors
}

\author{
Roman Sosnowski ${ }^{1}$, Małgorzata Benke², Tomasz Demkow' ${ }^{1}$,Marcin Ligaj³, Wojciech Michalski4 \\ 'Urooncology Department, Maria Skłodowska-Curie, Memorial Cancer Hospital, Warsaw, Poland \\ ${ }^{2}$ Nuclear Medicine Department, Maria Skłodowska-Curie, Memorial Cancer Hospital, Warsaw, Poland \\ ${ }^{3}$ Pathology Department, Maria Skłodowska-Curie, Memorial Cancer Hospital, Warsaw, Poland \\ ${ }^{4}$ Biostatistic Department, Maria Skłodowska-Curie, Memorial Cancer Hospital, Warsaw, Poland
}

\section{KEY WORDS}

kidney cancer nephron sparing surgery (NSS) D glomerular filtration rate (GFR)

\section{ABSTRACT}

\begin{abstract}
Nephron sparing surgery (NSS) is a technique more frequently utilized in the case of surgical treatment of kidney tumor. The aim of the study is to assess renal function in patients treated with NSS for renal tumors. Twenty patients, after NSS performed for renal tumor, were included in the study. In each patient dynamic renal scintigraphy was performed before surgical treatment (test No. 1) and after a mean interval of 12 months after surgical procedure (test No. 2). In each test renographic curves were evaluated. Creatinine levels and GFR rates were also assessed. Mean GFR was $84 \mathrm{ml}$ $\mathrm{min} / 1.73 \mathrm{~m}^{2}$ before surgery, and $79 \mathrm{ml} / \mathrm{min} / 1.73 \mathrm{~m}^{2}$ after surgical procedure. Mean change of GFR value after the surgical procedure was -5.1 ( $p>0.058$ ). On renography significant deterioration of renal function was observed in the operated kidneys of 4 patients (20\%) after NSS, insignificant deterioration - in four patients (20\%) and improvement of renal function of operated kidney was found in one patient. In 12 patients (60\%) no change was observed. The relative percentage GFR-share of operated kidney decreased by the average value of 3.8\% $(p>0.005)$. Multifactorial analysis did not identify significant effect of potential risk factors on the function of the kidney subjected to NSS. Preliminary results of this study confirm that deterioration of renal function after NSS is a rare event.
\end{abstract}

\section{INTRODUCTION}

Over the last decade significant advances in diagnosis and treatment of renal tumors were made mostly due to the universal access to imaging modalities like ultrasonography, computed tomography (CT) as well as magnetic resonance imaging (MRI). As a result, detection rate of incidental tumors (often less than $4 \mathrm{~cm}$ in diameter) and organ-confined tumors increased significantly [1]. In the past, radical nephrectomy (RN) was considered to be the "gold standard" in renal tumor treatment. However, it has been observed that with sufficient follow-up total unilateral nephrectomy can result in deterioration of kidney function as reflected e.g. by serum creatinine [2]. Nowadays, surgical procedures aiming at preservation of greatest possible volume of healthy kidney are increasingly performed. Partial kidney resection for tumor or nephron-sparing surgery (NSS) is mostly applied in the treatment of organ-confined renal cancer [3]. Initially, indications for NSS included: small tumors ( $<4 \mathrm{~cm}$ in diameter), impaired renal function, tumor in a solitary kidney or a tumor in the only functional kidney. With the improvement of surgical techniques NSS was applied in a wider range of patients including those with bigger tumors $(>4 \mathrm{~cm})$. At present, indications for NSS include tumors up to $7 \mathrm{~cm}$ in diameter and of any location. NSS is at present a validated treatment method with proven efficiency and safety. It was published that the incidence of chronic renal failure after radical nephrectomy is significantly higher than after NSS [4]. Lower baseline GFR in patients after surgical treatment for renal tumors is associated with reduced overall survival and patient mortality appears to be related to the decrease of GFR regardless of the cause of death $[5,6,7]$.

Our aim is to assess kidney function in patients treated with nephron-sparing surgery for renal tumors. In this paper we present preliminary results of our study performed in a group of patients treated in the Urooncology Department of Maria SkłodowskaCurie, Memorial Cancer Hospital in Warsaw, Poland.

\section{SUBJECTS AND METHODS}

Between January 2000 and September 2006, 106 nephronsparing surgeries were carried out in our Urooncology Department. Each patient was CT-scanned before the surgery. Furthermore, in all patients operated on before 2004 every NSS was preceded by intravenous pyelography. According to then introduced novel standard of pre-NSS patient preparation and care, renal scintigraphy was performed in all patients operated on in 2004 and onwards. In the years 2004-2006 preoperative renography was done in 29 patients undergoing NSS. This subgroup of 29 patients was evaluated prospectively and qualified for postoperative renal scintigraphy. This was done in 20 patients (where written consent was obtained), who were eventually included in our study. Mean NSS-renography interval was 12 months (range 6 to 46 months). Pre-operative scintigraphy (referred to below as test No. 1) and the post-operative scintigraphy (referred to as "test no. 2") were, in each patient, carried out under the same conditions with the use of Apex Helix Elscint - a two-head gamma camera. A dose of 259 $\mathrm{MBq}$ Tc $99 \mathrm{~m}$ DTPA was administered $/ V$ in order to assay the glomerular filtration rate (GFR). The raw acquired counts were analyzed with dedicated computer software. In each test renographic curve was assessed separately for each kidney and classified further to the respective 0 'Reilly category, with 0 and 1 values representing normal function, 2 - elongation of secretory phase, 3 - considerable elongation of secretory phase with unmarked excretory phase (cumulative curve), 4 - substantial function impairment (isosthenuric curve) (Fig. 1) [8].

The following parameters were then analyzed: GFR value $(\mathrm{ml})$ $\min / 1.73 \mathrm{~m}^{2}$ ), relative percentage GFR-share of each kidney, type of 


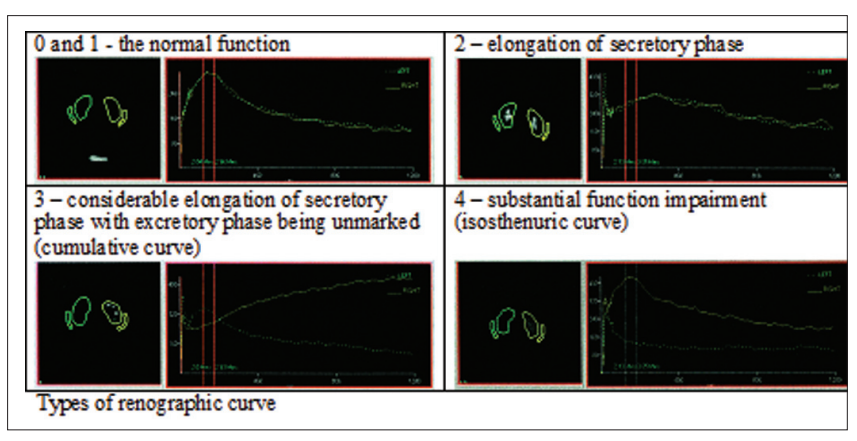

Fig. 1. Types of renographic curve.

renographic curve, patient age, histopathological diagnosis, tumor size and surgical margins (completeness of resection).

Change in the type of renographic curve accompanied by decrease of GFR was considered insignificant deterioration of operated kidney function. Change by at least two types of renographic curve with decrease of GFR was considered significant deterioration of the operated kidney and its function. Where GFR was subjected to an insignificant change and no switch the shape of the curve was noted, the operated kidney function was marked "unchanged".

Kidney function was assessed on the basis of GFR value. Supplementary serum creatinine measurements were performed, directly before NSS and approximately 12 months after the surgery.

We used the Student's pair t-test to compare the pre- and post- operative GFR and creatinine levels.

\section{RESULTS}

Mean patient age was 59 years (range 32-73 years). Histology for tumors resected by NSS was: renal cell carcinoma in 12 patients, renal oncocytoma in 4 patients, angiomyolipoma in 1 patient, papillary adenoma in 1 patient, and benign cystic lesions in 2 patients. Tumor stage was pT1a in 11 patients and pT1b in 1 remaining patients. Fuhrman grade of renal cell carcinoma cases $(n=12)$ was $\mathrm{G} 1$ in 2 cases, G2 in 7 cases, G3 in 3 cases.

Incomplete tumor resection (positive surgical margin) was seen in one patient. All patients were closely followed up for 6 to 46 months; including the patient with positive surgical margin (follow-up time of 15 months). No local recurrence or tumor dissemination was found in any of the patients.

Mean GFR before surgery (test No. 1) was $84 \mathrm{ml} / \mathrm{min} / 1.73 \mathrm{~m}^{2}$. Mean GFR after NSS (test no 2) was $79 \mathrm{ml} / \mathrm{min} / 1.73 \mathrm{~m}^{2}$. Mean change of GFR value after the surgical treatment was - 5.1 ( $p$ $>0.058$ ). Mean serum creatinine before NSS was 0.91 (range $0.6-2.45$ ). Mean postoperative serum creatinine was 1.08 (range 0.8-2.58). In one patient severe deterioration of kidney function appeared secondary to chronic renal cirrhosis (contralateral kidney with no tumor) (pre-NSS creatinine - 0.9, post-NSS - 2.45). Significant statistical difference was observed between the levels of serum creatinine before and after NSS ( $p<0.001)$. Comparison of renography results (test no. 1 vs. test no. 2) identified 4 patients (20\%) with significant deterioration of renal function after NSS (GFR decrease by a mean value of 17.1 and switch in the renographic curve type: from type 1 or 2 to type 4). No cases of kidney failure were diagnosed after NSS as specified by KDOOI criteria [9]. Insignificant deterioration of function of operated kidney was observed in four patients (20\%) and improvement of renal function of operated kidney was found in one patient. Renal function after NSS in the remaining 12 patients (60\%) remained unchanged after NSS. The relative percentage share of operated kidney decreased by

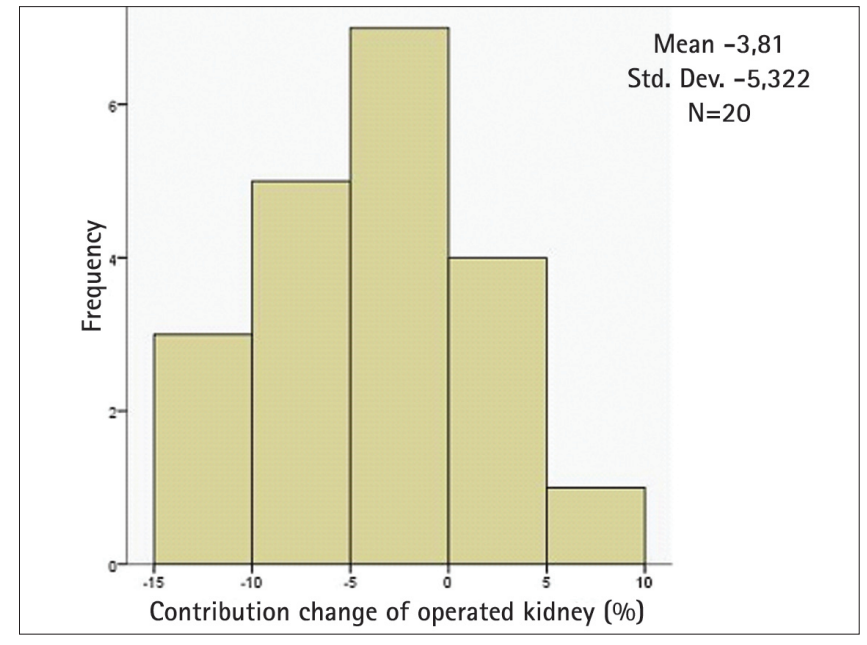

Fig. 2. Difference of the relative percentage share of operated kidney.

a mean value of 3.8\% ( $p>0.005$ ) (Fig. 2). The following risk factors potentially affecting kidney function were identified in patients' medical history: nicotinism - in 2 patients, hypertension - 9 patients, diabetes - 1 patient, and prior chemotherapy - 1 patient. Due to a relatively small study group statistical analysis of potential influence of risk factors on kidney function was not possible.

\section{DISCUSSION}

Application of surgical techniques, like nephron-sparing surgery, that aim at sparing greatest possible volume of healthy parenchyma of operated organs is increasingly important in tumor surgery. They seem to render comparable results of long-term tumor-related survival [10]. In the past, the principle of sparing greatest possible volume of healthy renal parenchyma of was not prioritized. This resulted from the assumption that the contralateral kidney (manifesting compensatory renal hypertrophy) would "take over" the function of the resected organ. However, in their research carried out on rats Hostetter et al. proved that resection of significant part of parenchyma caused renal failure of the operated kidney [11]. Hostetter designed a classic set of experiments in a rat to confirm that extensive ablation of renal tissue leads to deterioration of renal function. However, this model involved unilateral total nephrectomy and a five-sixth nephrectomy of the contralateral kidney; it is not clear whether unilateral nephrectomy ablates a sufficient quantity of tissue to contribute to subsequent renal damage in humans. There are no clear criteria for determination of chronic renal failure. Serum creatinine level is one of the parameters, taken into account, but no clearly defined threshold is used. Some authors diagnose chronic kidney failure with serum creatinine $>1.5 \mathrm{mg} / \mathrm{dL}$ whereas others with levels $>2.0 \mathrm{mg} / \mathrm{dL}[12,13]$. Another way of chronic kidney disease assessment is the determination of GFR with glomerular filtration rate value below $60 \mathrm{ml} / \mathrm{min} / 1.73 \mathrm{~m}^{2}$ consistent with kidney failure [9]. In our study we used both serum creatinine and GFR as parameters reflecting renal function. Najarian et al. analyzed patients subjected to donor nephrectomy for transplantation and noted that with follow-up of 20 years mean serum creatinine level was $1.1 \mathrm{mg} / \mathrm{dL}$ and glomerular filtration rate decreased by $20 \%$ as compared with these parameters before surgical treatment [14]. Mean age of kidney donors in that group was 43 years (as compared with mean age of 59 years in our group) and renal cancer risk factors such as nicotinism, hypertension, and diabetes rarely occur in kidney donors. Wishnow et al. studied patients subjected to radical nephrectomy and noted that after 12 years of 
follow-up after surgery few patients showed abnormal elevation of serum creatinine [2]. While analyzing relation and incidence of chronic renal failure after radical nephrectomy (RN) or NSS Lau et al. observed relatively lower cumulative incidence of chronic renal failure in patients subjected to NSS as compared with patients after RN (11.6\% vs. 22.4\% respectively, relative risk 3.7) [4]. Haung et al. noted significant differences in occurrence of moderate and severe chronic renal failure in a group of patients treated with RN or NSS for renal tumors less than $4 \mathrm{~cm}$ in diameter. Mean time between surgery and kidney failure with GFR $<60 \mathrm{~mL} / \mathrm{min} 1.73 / \mathrm{m}^{2}$ in patients after RN was 18 months, while no cases of chronic kidney failure occurred in patients after NSS [15]. In our study, with significantly shorter follow-up, we identified only one patient (5\%) with a normal contralateral kidney and chronic renal failure after NSS. However, we used minimal serum creatinine level of $1.5 \mathrm{mg} / \mathrm{dL}$ as a value defining chronic renal failure. Pettus et al. studied a group of 1479 patients treated for renal tumor with NSS or RN with follow-up for 10 years. In this study lower baseline GFR was associated with reduced overall survival [5]. In the study of over 1 million patients, Go et al. found that patient mortality was related to the decrease of GFR regardless of the cause of death [6]. Risk factors such as diabetes, ischemic heart disease, stroke and other coexisting diseases were analyzed. The increased risk of death was associated with the decrease of GFR value below 60 $\mathrm{ml} / \mathrm{min} / 1.73 \mathrm{~m}^{2}$. Hazard ratio for death for GFR ranges of $45-59$, $30-44,15-29$ and below $15 \mathrm{ml} / \mathrm{min} / 1.73 \mathrm{~m}^{2}$ was $1.2 ; 1.8 ; 3.2 ; 5.9$, respectively ( $95 \% \mathrm{Cl} 5.4$ to 6.5$)$.

In our study group kidney function after NSS for renal tumor deteriorated significantly in 20\% of patients as evidenced by GFR decrease and renography results. The results of this study show that significant renal function impairment after this type of surgical procedure is a relatively rare event, which is in concordance with the published data. With longer follow-up we will attempt to assess NSS-related renal function dynamics and its potential effect on survival.

\section{REFERENCES}

1. Konnak JW, Grossman HB: Renal cell carcinoma as an incidental finding. J Urol 1990; 134: 1094-1096.

2. Wishnow $\mathrm{KI}$, Johnson $\mathrm{DE}$, Preston $\mathrm{D}$ : Long-term serum creatinine values after radical nephrectomy. Urology 1990; 35: 114-116.

3. Lee CT, Katz J, Shi W: Surgical management of renal tumors $4 \mathrm{~cm}$ or less in a contemporary cohort. J Urol 2000; 163: 730-736.

4. Lau WK, Blute ML, Weaver AL: Matched comparison of radical nephrectomy vs. nephron-sparing surgery in patients with unilateral renal cell carcinoma and a normal contralateral kidney. Mayo Clin Proc 2000; 75: 1236-1242.

5. Pettus JA, Janq TL: Effect of baseline glomerular filtration rate on survival in patients undergoing partial or radical nephrectomy for renal cortical tumors. Mayo Clin Proc 2008; 83: 1101-1106

6. Go AS, Chertow GM, Fan D: Chronic kidney disease and the risks of death, cardiovascular events, and hospitalization. Engl J Med 2004; 351: 12961305.

7. Fried LF, Katz R, Sarnak MJ: Kidney function as a predictor of non cardiovascular mortality. J Am Soc Nephrol 2005; 16: 3728-3735.

8. O'Reilly P, Aurell M, Britton K: Consensus on diuresis renography for investigating the dilated upper urinary tract: Radionuclides in Nephrourology Group - Consensus Committee on Diuresis Renography. J Nucl Med 1996; 37: 1872-1876.

9. Work Group KDOOI Clinical Practice Guidelines for Chronic Kidney Disease: Evaluation, Classification, and Stratification, 2002 National Kidney Foundation http://www.kidney.org/professionals/KDOQI/index.cfm

10. Uzzo RG, Novick AC: Nephron sparing surgery for renal tumors: Indications, techniques and outcomes. J Urol 2001; 166: 6-18.
11. Hostetter TH, Olson JL, Rennke HG: Hyperfiltration in remnant nephrons: a potentially adverse response to renal ablation. Am J Physiol 1981; 241: 85-93.

12. Hsu CY, Chertow GM: Chronic renal confusion: insufficiency, failure, dysfunction, or disease. Am J Kidney Dis 2000; 36: 415-418.

13. McKiernan J, Simmons R, Katz J, Russo P: Natural history of chronic renal insufficiency after partial and radical nephrectomy. Urology 2002; 59: 816-820.

14. Najarian JS, Chavers BM, McHugh LE: 20 years or more of follow-up of living kidney donors. Lancet 1992; 340: 807-810.

15. Huang WC, Levey AS, Serio AM: Chronic kidney disease after nephrectomy in patients with renal cortical tumors: a retrospective cohort study. Lancet Oncol 2006; 7: 735-740.

\section{Correspondence}

Roman Sosnowski Urooncology Department Cancer Memorial Hospital 5, Roentgena Street 02-781 Warsaw, Poland phone: +48225462000 roman.sosnowski@gmail.com 